\title{
Effect of Au Addition on the Crystallinity and Electrical Properties of Thermally Strained $\mathrm{LaNiO}_{3-\delta}$ Thin Films
}

\author{
M.W. ZHU, ${ }^{1}$ D.C. SHI, ${ }^{2}$ X. ZHAO,${ }^{2}$ N. JIA,${ }^{2}$ and C.Z. LIU $^{1,3}$ \\ 1.-School of Material Science and Engineering, Shenyang Aerospace University, Shenyang \\ 110136, People's Republic of China. 2.-Key Laboratory for Anisotropy and Texture of Materials \\ (ATM), Northeastern University, Shenyang 110819, People's Republic of China. 3.-e-mail: \\ czliu@sau.edu.cn
}

\begin{abstract}
We prepared $\mathrm{Au}-\mathrm{LaNiO}_{3-\delta}(\mathrm{Au}-\mathrm{LNO})$ nanocomposite thin films by a sol-gel coating method. The effects of the doped Au on the microstructure and electrical properties of the Au-LNO films under different types of thermal strain were investigated. Introduction of Au into the LNO films changed their preferential orientation and markedly lowered their room-temperature resistivity. Moreover, the resistivity of the films containing $\mathrm{Au}$ nanoparticles showed negligible dependence on thermal strain, in contrast to the behavior of $\mathrm{Au}$-free LNO films. We performed microstrain analysis and x-ray photoelectron spectroscopy to explore the strain-resistivity relationship of the films. Microstrain was closely related to the density of oxygen vacancies in LNO films and dominated the change in electrical properties of polycrystalline LNO films. The addition of Au promoted crystallization of the LNO films and decreased the density of oxygen vacancies, which stabilized the microstrain in the films under different external strain and accounted for the strain independence of the resistivity of the Au-LNO films.
\end{abstract}

Key words: Conductive oxide, sol-gel process, nanocomposite film, microstrain, oxygen vacancy

\section{INTRODUCTION}

Lanthanum nickel oxide $\left(\mathrm{LaNiO}_{3-\delta}, \mathrm{LNO}\right)$ is a strongly correlated perovskite oxide with excellent physical properties, including high conductivity, thermoelectricity, and potential to achieve superconductivity. ${ }^{1-3}$ The metallic conductivity and chemical stability of LNO films make them suitable for use as electrodes for high-quality dielectric and ferroelectric materials in electronic devices. ${ }^{4,5}$ Extensive investigations on LNO thin films have been conducted in recent years, particularly on methods to lower the resistivity of LNO films. The resistivity of LNO films may be lowered by depositing high-quality single crystal films or synthesizing nanocomposite films with highly conductive metals. ${ }^{6,7}$

(Received December 15, 2018; accepted July 18, 2019;

published online July 29, 2019)
The effects of strain are unavoidable in singlecrystal and nanocomposite films. The influence of strain on the electrical properties of epitaxial LNO films has been intensively investigated.8,9 Strain plays an important role in improving the conductivity of LNO films, because the Ni-O-Ni bond angle and length change when the films are in different strain states. ${ }^{10}$ Thus, the electrical properties of LNO films are determined by their substrates, which can exert different types of strain; i.e., epitaxial and thermal strain. For polycrystalline ferroelectric films, silicon ( $\mathrm{Si}$ ) is most often used as a substrate in electronic devices, and tensile thermal strain existing in the films contributes to their ferroelectric properties. ${ }^{11-13}$ However, for LNO films, previous investigations have shown that tensile strain has a negative effect on their conductivity. ${ }^{14,15}$ Accordingly, the working voltage and power dissipation increase in devices in which an LNO film is used as the bottom electrode on an $\mathrm{Si}$ substrate. Therefore, it is necessary to alleviate the 
adverse effect of tensile strain on the electrical properties of LNO films.

The effects of strain on resistivity can be adjusted by controlling the composition of epitaxial LNO films. ${ }^{16}$ Upon introducing excess nickel element $(\mathrm{Ni})$ into epitaxial LNO films, the resistivity of LNO sharply decreases under the same tensile strain. One possible underlying mechanism for this behavior is the increase of defect density under strain; thus, it may be possible to tailor the effects of strain by controlling cation stoichiometry and the type of defects in films. ${ }^{17,18}$ For composite films, the second phase affects the crystallization process and the distribution of strain within the matrix phase. ${ }^{19,20}$ However, it remains unclear how LNO films might behave under a strain field upon introduction of a second phase and whether the unfavorable effects of tensile strain be mitigated. In this paper, we prepare $\mathrm{Au}-\mathrm{LaNiO}_{3-\delta}$ (Au-LNO) nanocomposite films in different strain states on different substrates by a sol-gel method. The effects of $\mathrm{Au}$ addition on the microstructures and electrical properties of the thermally strained Au-LNO films are explored. By comparison with the behavior of $\mathrm{Au}-$ free LNO films, the strain dependence of the electrical properties of Au-LNO films is systematically investigated and a mechanism for the anomalous strain-dependent resistivity of Au-LNO films is proposed.

\section{EXPERIMENTAL PROCEDURE}

\section{Preparation and Characterization}

A precursor solution for the LNO coating was prepared from lanthanum nitrate $\left[\mathrm{La}\left(\mathrm{NO}_{3}\right)_{3} \cdot 6 \mathrm{H}_{2} \mathrm{O}\right]$, nickel acetate $\left[\mathrm{Ni}\left(\mathrm{CH}_{3} \mathrm{COO}\right)_{2} \cdot 4 \mathrm{H}_{2} \mathrm{O}\right], 2$-methoxyethanol $\left(\mathrm{CH}_{3} \mathrm{OC}_{2} \mathrm{H}_{4} \mathrm{OH}\right)$, and acetic acid (AC, $\mathrm{CH}_{3} \mathrm{COOH}$ ). A certain amount of hydrogen tetrachloroaurate $\left(\mathrm{HAuCl}_{4} \cdot 3.5 \mathrm{H}_{2} \mathrm{O}\right)$ was added to the above precursor to obtain a solution with an $\mathrm{Au}$ content of 2.38 at.\%. The clear and stable precursor solutions with a concentration of $0.10 \mathrm{M}$ were aged for $24 \mathrm{~h}$. The solutions were then coated onto substrates with different coefficients of thermal expansion (CTE; LNO: $8.6 \times 10^{-6} /{ }^{\circ} \mathrm{C}, \quad \mathrm{Si}$ : $3.5 \times 10^{-6} /{ }^{\circ} \mathrm{C}, \quad \mathrm{Al}_{2} \mathrm{O}_{3}: \quad 7.5 \times 10^{-6} /{ }^{\circ} \mathrm{C}, \quad \mathrm{MgO}$ : $11 \times 10^{-6} /{ }^{\circ} \mathrm{C}$ ). Coating was performed on a spin coater operated at $1000 \mathrm{rpm}$ for $2 \mathrm{~s}$ and then at $4000 \mathrm{rpm}$ for $50 \mathrm{~s}$. Amorphous $\mathrm{Al}_{2} \mathrm{O}_{3}$ films with a thickness of $60 \mathrm{~nm}$ were deposited on all the substrates by magnetron sputtering, to guarantee the same surface condition of the substrates for the subsequent deposition of LNO and Au-LNO films. The coated films were dried at $120^{\circ} \mathrm{C}$ for $10 \mathrm{~min}$ on a hot plate and then annealed in a furnace at $650^{\circ} \mathrm{C}$ for $30 \mathrm{~min}$. Finally, the films were removed from the furnace and cooled in air. This process was repeated eight times to obtain the desired film thickness. The cation ratio of $\mathrm{Ni}$ to $\mathrm{La}$ was precisely controlled to $1: 1$ in the precursor solution, and the resulting films should be stoichiometric; i.e., indexed as $\mathrm{LaNiO}_{3-\delta}$.
The crystal structure and preferred orientations of the films were analyzed by x-ray diffraction (XRD; Rigaku RINT2000, $\mathrm{Cu} \mathrm{K} \alpha$ radiation). The surface morphologies and cross-sectional microstructures of the films were observed by scanning electron microscopy (SEM; Ultra Plus). We performed scanning transmission electron microscopy-high angle annular dark field (STEMHAADF) measurements to obtain the distribution of $\mathrm{Au}$ in the LNO films with a transmission electron microscope (JEOL JEM-2100F). The valence states of oxygen in the films were determined by x-ray photoelectron spectroscopy (XPS; RIBER LAS-3000) with monochromatic $\mathrm{Al} \mathrm{K} \alpha$ radiation with a photon energy of $1486.6 \mathrm{eV}$. Binding energies were calibrated to the $\mathrm{C} 1 \mathrm{~s}$ binding energy of $284.6 \mathrm{eV}$. The resistivity of the LNO films was measured with the standard four-point probe method (RTS-11, Four Probes Tech).

\section{Microstrain Calculation}

In principle, the experimental XRD profile is a convolution of the instrumental profile and the intrinsic profile, which can be approximated by the Cauchy or Gaussian function. The William-Hall method is usually adopted to determine the values of strain broadening and grain size when they are both represented by the Cauchy function, ${ }^{21}$ as follows:

$$
\frac{\beta \cos \theta}{\lambda}=\frac{1}{D}+4 \varepsilon \frac{\sin \theta}{\lambda}
$$

Klug and Alexander suggested an alternative method, proposing that the strain broadening and effect of crystallite size could be better approximated by Gaussian and Cauchy functions, respectively, ${ }^{22,23}$ through the following relation:

$$
\frac{\beta^{2}}{\operatorname{tg}^{2} \theta}=\frac{\lambda}{D}\left(\frac{\beta}{\operatorname{tg} \theta \sin \theta}\right)+16 \varepsilon^{2}
$$

where $D$ is the average grain size, $\varepsilon$ is microstrain, and $\theta, \beta$, and $\lambda$ denote the same parameters as used in the Scherrer formula. To determine the microstrain of Au-free LNO and Au-LNO films under external thermal strains, we measured the XRD line profiles of (100) or (110) reflections in combination with those of (200) reflections for the films deposited on different substrates using an X'pert MRD diffractometer with a $\mathrm{Cu} \mathrm{K} \alpha$ radiation source. Diffraction data were collected by scanning each $h k l$ reflection with a step size of $0.01^{\circ}$.

\section{RESULTS AND DISCUSSION}

\section{Microstructure and Orientation of Au-LNO Films}

The microstructures of Au-LNO films deposited on different substrates were examined by SEM, as 
shown in Fig. 1. In all cases, minor changes in grain size were observed for the films deposited on different substrates. Grain sizes in the films showed a homogeneous distribution when the white grains were excluded. Thus, strain had minimal effect on the grain growth of LNO in the current case. Crosssectional observations showed that compact films with a thickness of $150 \mathrm{~nm}$ formed, and no microcracks or hillocks were identified. Thus, the introduction of Au promoted crystallization of LNO films and facilitated growth of columnar structures.

The distribution of $\mathrm{Au}$ in the films was examined by STEM to understand its effect on the crystallization of the LNO films. Both $\mathrm{La}$ and $\mathrm{Ni}$ were homogenously distributed throughout the films, and the white grains dispersed in the films were confirmed to be $\mathrm{Au}$ (Fig. 2). The grain size of the LNO matrix was generally uniform, with an average value of $\sim 45 \mathrm{~nm}$. Conversely, the secondary phase $\mathrm{Au}$ ) was classified into two categories, i.e., large $\mathrm{Au}$ grains that mainly existed at LNO grain boundaries and small Au grains within LNO grains, as shown in Fig. $2 \mathrm{e}$ and $\mathrm{f}$, respectively. We propose that the difference in grain sizes was determined by their crystallization processes. These two types of $\mathrm{Au}$ grains have different effects on the grain growth of the Au-LNO composite films and consequently, on their electrical properties. Smaller Au grains $(\sim 10 \mathrm{~nm})$ grew before the annealing process and provided nucleation sites that promoted the crystallization of LNO; this effect improved the crystallinity of the films. Conversely, larger $\mathrm{Au}$ grains $(30-40 \mathrm{~nm})$ distributed at the LNO grain boundaries could form simultaneously with crystallization of LNO grains. Although these Au grains had negligible effect on the nucleation process of LNO grains, the Au grains might promote the formation of conducting pathways. ${ }^{24}$

Figure 3a shows the XRD patterns of the Au-LNO films deposited on different substrates. Only peaks from $\mathrm{LaNiO}_{3}$ and $\mathrm{Au}$ were identified, indicating that the films were well crystallized as a single perovskite phase with no impurity phases except for $\mathrm{Au}$. The Au-LNO films showed a preferential (110) orientation because of the introduction of $\mathrm{Au}$, whereas the (100) peak dominated the diffraction pattern of the Au-free LNO film (inset of Fig. 3a). Usually, the preferred orientation of a film strongly depends on the early stages of nucleation and growth. The above results confirmed that the presence of $\mathrm{Au}$ affected the crystallization process of LNO grains. Furthermore, the LNO diffraction peaks shifted when different substrates were used, as shown in Fig. 3b and Table I. The peak shift was attributed to thermal strain caused by the mismatch between the CTEs of the LNO films and substrates, defined as $\varepsilon_{\text {thermal }}=\left(\alpha_{f}-\alpha_{s}\right)\left(T_{2}-T_{1}\right)$ (in which $\alpha_{\mathrm{f}}$ and $\alpha_{\mathrm{s}}$ are CTEs of the film and the substrate, $\mathrm{T}_{1}$ is room temperature, and $\mathrm{T}_{2}$ is annealing temperature). Here, compressive and tensile strains were exerted on the Au-LNO films through the use of substrates with appropriate
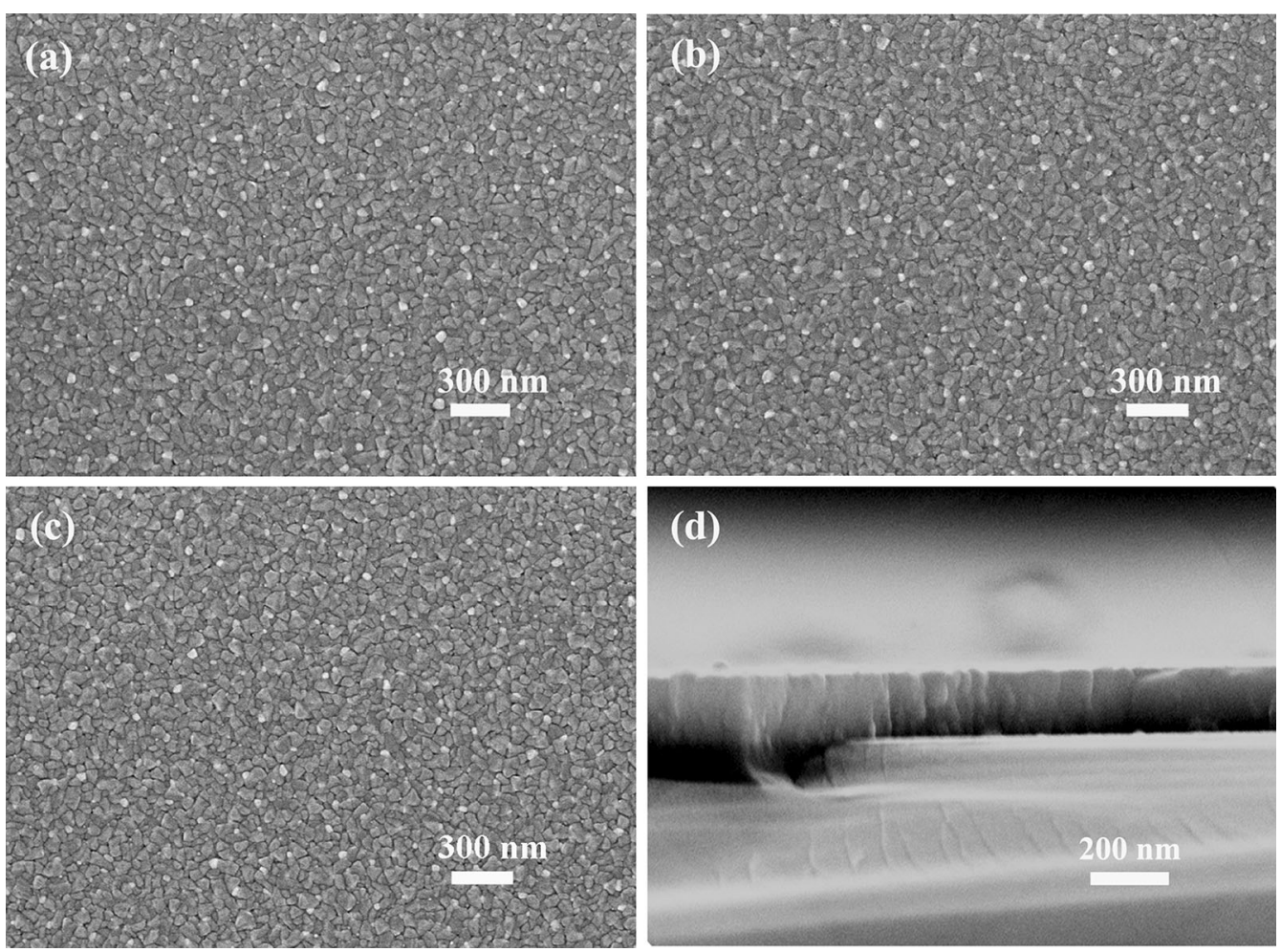

Fig. 1. Surface morphologies of Au-LNO on (a) $\mathrm{MgO}$, (b) $\mathrm{Al}_{2} \mathrm{O}_{3}$, and (c) Si substrates, and (d) corresponding cross-sectional structure of an AuLNO film deposited on Si. 

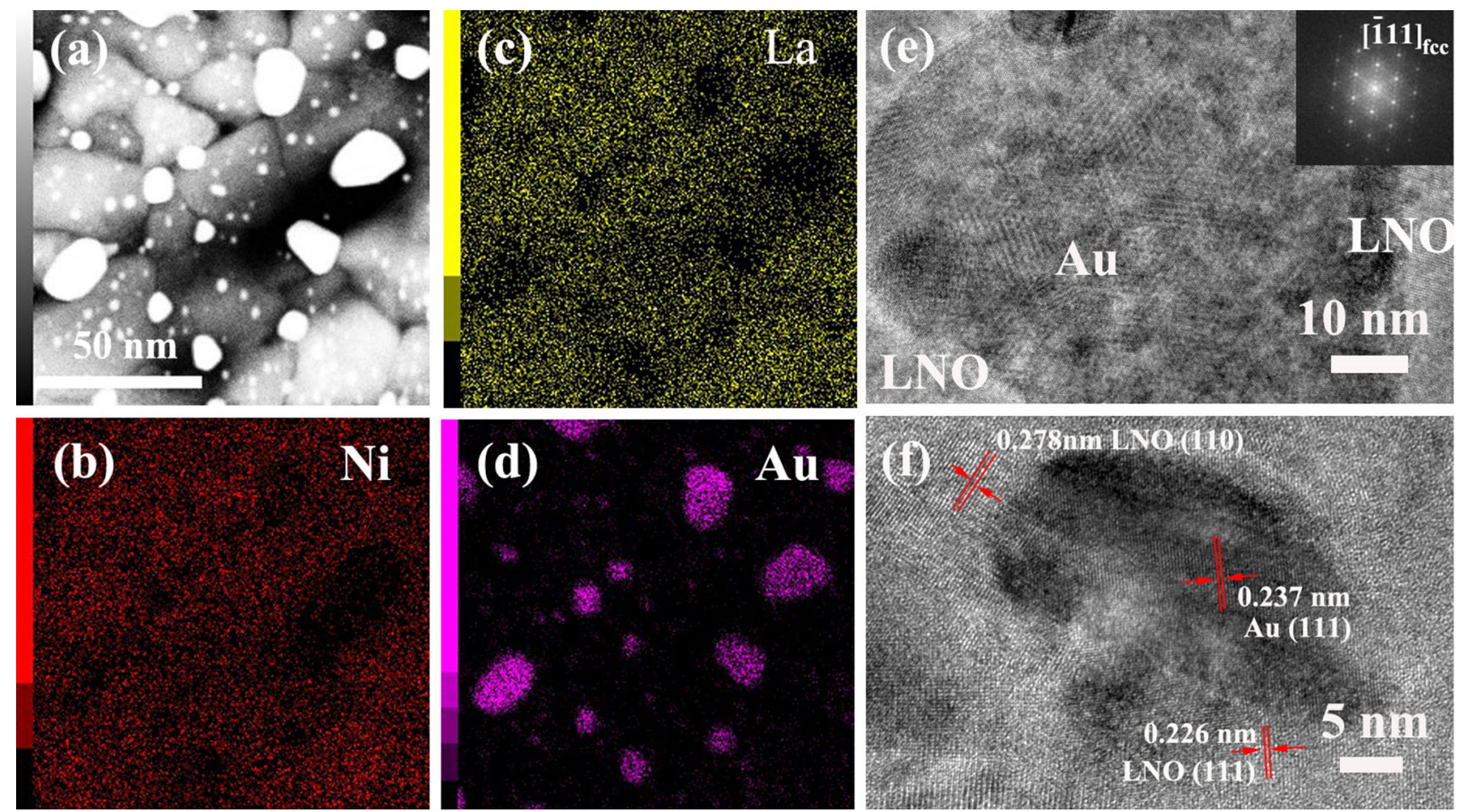

Fig. 2. (a) STEM surface morphology of an Au-LNO thin film on Si substrate. Element mapping of (b) La, (c) Ni, and (d) Au. HRTEM images of an Au-LNO film showing (e) a large Au particle at an LNO grain boundary and (f) a small Au particle in an LNO grain.
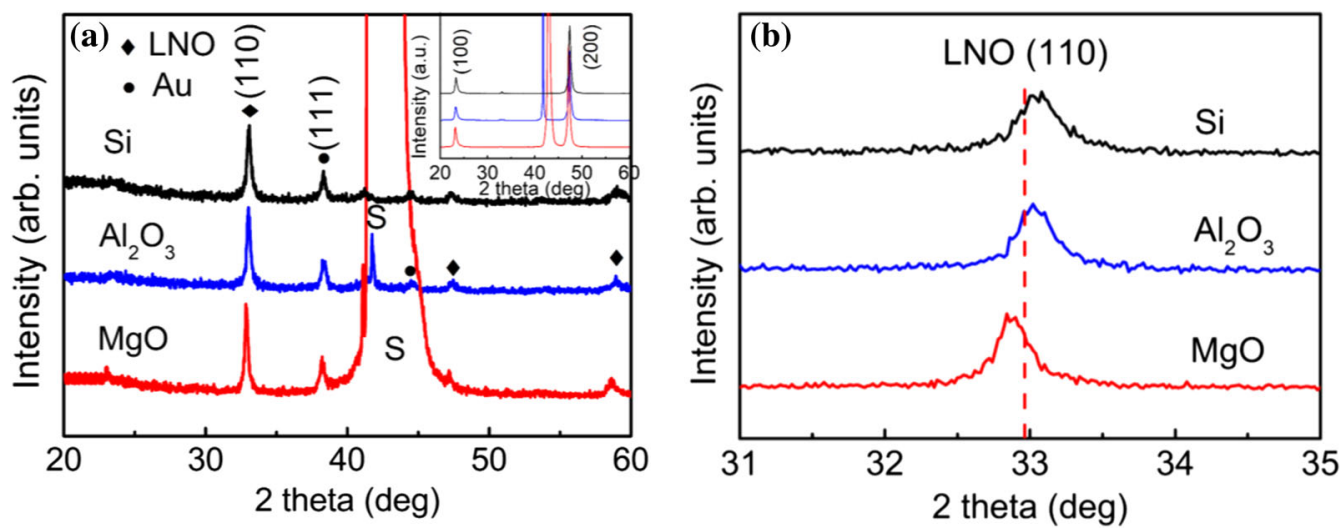

Fig. 3. (a) XRD patterns of $\mathrm{Au}$-LNO films on $\mathrm{Si}, \mathrm{Al}_{2} \mathrm{O}_{3}$, and $\mathrm{MgO}$ substrates and (b) enlarged view of the region from $31^{\circ}$ to $35^{\circ}$. The inset shows the XRD patterns of Au-free LNO films on the same substrates.

Table I. Lattice parameters of the Au-LNO films on different substrates

\begin{tabular}{lcccc}
\hline Substrate & $\mathbf{M g O}$ & $\mathbf{A l}_{\mathbf{2}} \mathbf{O}_{\mathbf{3}}$ & \multicolumn{1}{c}{$\mathbf{S i}$} \\
Theoretical thermal strain & $-0.29 \%$ & $0.077 \%$ & $0.357 \%$ \\
Orientation & $(110)$ & $(110)$ & $(110)$ \\
$a_{\perp}(\AA)^{\mathrm{a}}$ & $3.849(3.843)$ & $3.832(3.828)$ & $3.829(3.823)$ \\
$a_{\|}(\AA)^{\mathrm{b}}$ & $3.835(-0.13 \%)$ & $3.844(0.10 \%)$ & $3.846(0.16 \%)$ \\
\hline
\end{tabular}

${ }^{\mathrm{a}}$ Values in parentheses are out-of-plane lattice constants of Au-free LNO films on the same substrate. ${ }^{\mathrm{b}}$ In-plane lattice constants were obtained based on the constant unit cell volume model, and the values in parentheses are the in-plane strain based on the change in lattice constant $\left[\delta=\left(a_{\|}-a_{b}\right) / a_{b}\right.$, where $a_{b}$ is the lattice constant of bulk LNO]. 
CTEs. The Au grains in the LNO films did not release strain, despite the differences of the CTEs and Young's moduli of Au and LNO. Thus, Au had a minor effect on the magnitude of thermal strain within the films.

\section{Electrical Properties of Au-LNO Films}

Figure 4a shows the room-temperature resistivity of Au-LNO films under different strain. For comparison, the resistivity of Au-free LNO films in the same strain states is also presented. As expected, the resistivity of the LNO films increased when the strain state changed from compressive to tensile, consistent with tendencies observed in other work. ${ }^{25}$ However, unlike that of the Au-free LNO films, the resistivity of the Au-LNO films was independent of the strain state. Thus, introduction of $\mathrm{Au}$ not only decreased the resistivity of the AuLNO films but also alleviated the dependence of the electrical properties on strain. The minimum resistivity of $383 \mu \Omega \mathrm{cm}$ was achieved for the Au-LNO film on Si. This value is comparable to that of films formed by physical vapor deposition. According to percolation theory, the resistivity of Au-LNO films on different substrates can also be calculated using Eq. 3, as shown in Fig. 4.

$$
\lg \rho_{\mathrm{Au}-\mathrm{LNO}}=\left(1-V_{\mathrm{Au}}\right) \lg \rho_{\mathrm{LNO}}+V_{\mathrm{Au}} \lg \rho_{\mathrm{Au}}
$$

From Fig. 4 and Table II, the calculated resistivity of the Au-LNO films was higher than that obtained experimentally. The difference between
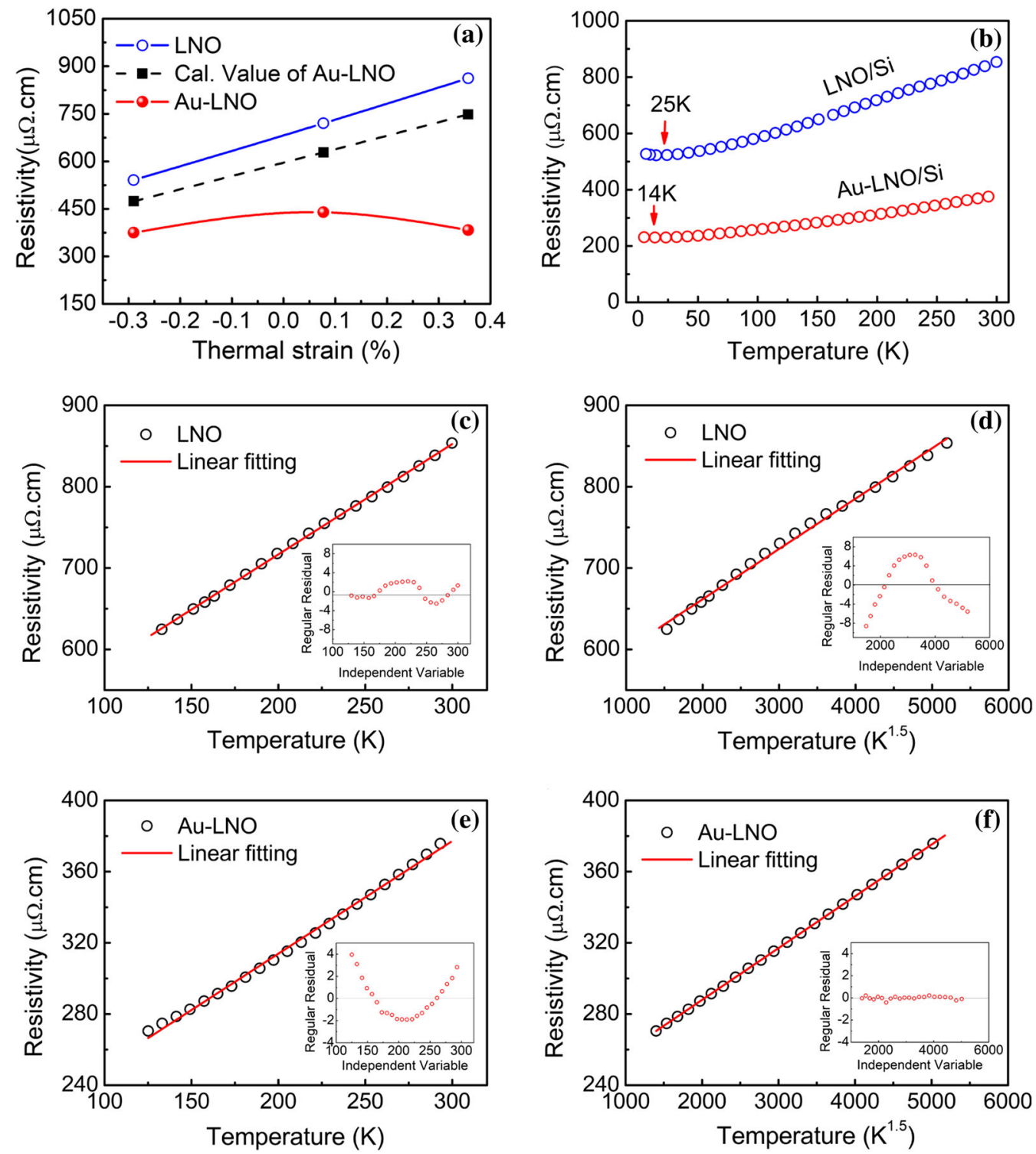

Fig. 4. (a) Resistivity of Au-LNO composite films on different substrates, (b) $\rho-T$ curve of LNO and Au-LNO films on Si substrates (every fifth data point is shown for clarity) and (c)-(f) linear $(\rho \propto T)$ and non-linear $\left(\rho \propto T^{1.5}\right)$ fitting results of the $\rho-T$ curves in (b). Insets show the fitting errors as a function of temperature. 
Effect of Au Addition on the Crystallinity and Electrical Properties of Thermally Strained $\mathrm{LaNiO}_{3-\delta}$ Thin Films

Table II. Room-temperature resistivity of LNO and Au-LNO films on different substrates

\begin{tabular}{|c|c|c|c|c|}
\hline Substrate & $\rho_{\mathrm{LNO}}(\exp ).(\mu \Omega \mathrm{cm})$ & $\rho_{\text {Au-LNO }}(\operatorname{exp.})(\mu \Omega \mathrm{cm})$ & $\left(\rho_{\text {Au-LNO }}-\rho_{\text {LNO }}\right) / \rho_{\text {LNO }}(\%)$ & $\rho_{\text {Au-LNO }}(\text { cal. })^{a}(\mu \Omega \mathrm{cm})$ \\
\hline $\mathrm{MgO}$ & 541 & 375 & -30.5 & 474.2 \\
\hline $\mathrm{Al}_{2} \mathrm{O}_{3}$ & 720 & 439 & -39.1 & 628.1 \\
\hline $\mathrm{Si}$ & 862 & 383 & -55.6 & 748.2 \\
\hline
\end{tabular}

${ }^{a}$ Volume and resistivity of $\mathrm{Au}$ in Eq. 3 are taken as $V_{\mathrm{Au}} \%=2.36 \%$ and $\rho_{\mathrm{Au}}=2.24 \mu \Omega . \mathrm{cm}$, and values of $\rho_{\mathrm{LNO}}$ in Eq. 3 are taken as the values of $\mathrm{Au}$-free LNO films on the same substrate as Au-LNO films.

the calculated and measured values suggests that the effect of added Au on the resistivity of LNO films cannot be simply evaluated as the summation of the resistivities of $\mathrm{Au}$ and $\mathrm{LNO}$. The above analysis indicates that the presence of $\mathrm{Au}$ can lower the resistivity of LNO by improving its crystallinity rather than through a simple mixing effect. Therefore, we concluded that the presence of Au affected some intrinsic changes in the Ni-O-Ni bond length/ angle or the variation of $\mathrm{Ni}$ valence induced by external strain, which might influence the strength of Ni-O hybridization.

We measured the transport properties of the LNO and Au-LNO films under tensile strain to better understand the effect of $\mathrm{Au}$ addition on film characteristics. From the resistivity-temperature ( $\rho-$ $T$ ) curves in Fig. 4b, the metal-insulator transition (MIT) temperature decreased from $25 \mathrm{~K}$ for the LNO films to $14 \mathrm{~K}$ for the Au-LNO films, indicating strengthening of Ni-O hybridization upon Au introduction. Moreover, in the metallic region (120$300 \mathrm{~K}$ ), a linear relationship between $\rho$ and $T$ was observed for the LNO films, which indicates that electron-phonon scattering dominates the transport process (as shown in Figs. 4c and d). In contrast, a large deviation was observed when the curve for the $\mathrm{Au}-\mathrm{LNO}$ films was linearly fitted, as shown in Fig. 4e. Instead, the resistivity was well fitted by non-linear fitting with a $T^{1.5}$ dependence. The new scattering behavior cannot be simply attributed to the combined effects of electron-electron scattering and electron-phonon scattering. Thus, the scattering mechanism is unlike Fermi liquid behavior. The deviation of Fermi liquid theory in the present case reflects changes induced by the introduction of $\mathrm{Au}$, ruling out the effects of strain. Namely, the deviation may originate from changes in the stoichiometry of the LNO films (related to defects such as oxygen vacancies or cation deficiency). ${ }^{16,26}$ Nonlinear behavior has been observed for single-crystalline $\mathrm{LaNiO}_{3}$, which has been attributed to its intrinsic quantum behavior. ${ }^{27} \mathrm{~A}$ transition from linear to nonlinear behavior was observed and was associated with the oxygen stoichiometry; a smaller amount of oxygen vacancies favored a $T^{1.5}$ dependence of the resistivity. ${ }^{28}$ In the present work, the $\mathrm{Ni} / \mathrm{La}$ ratio was accurately controlled at the atomic level. No volatilization of elements should be expected at the current annealing temperature; therefore, our films were stoichiometric in terms of cations. Thus, the deviation of the transport behavior is likely caused by a decrease in the density of oxygen vacancies in the Au-LNO films compared with that in the LNO films.

\section{Microstrain Analysis of Au-LNO Films}

For polycrystalline films, normally, two effects are expected under biaxial external strain: first, changes of the peak positions and lattice constants are directly influenced by macrostrain, as discussed above. Second, widening of diffraction peaks induced by microstrain is closely related to the formation of defects in the bulk of the films. ${ }^{29}$ Direct changes of the lattice constants affect the Ni-O-Ni bond angle and length, whereas changes in the defect density cause variation of the element valence and $\mathrm{Ni}-\mathrm{O}-\mathrm{Ni}$ bond parameters. The effects of macrostrain dominate epitaxial LNO films and change their resistivity in different strain states. 8,10 For polycrystalline LNO and Au-LNO films, different trends were observed for resistivity with varying strain states, although the effect of $\mathrm{Au}$ on macrostrain was negligible. Therefore, the effects of microstrain on the resistivity should be emphasized.

To clearly understand the relationship between the strain and electrical properties of Au-LNO films, the XRD peaks of each film were further analyzed. The average grain size and microstrain of the films were calculated from the XRD measurements using the William-Hall (Eq. 1) and Klug-Alexander (Eq. 2) methods. The validity of these methods was confirmed by comparing the calculated grain size with that obtained from SEM to STEM results. The microstrain in the LNO films increased sharply when the thermal strain state changed from compressive to tensile (Fig. 5a). However, little change was observed in the microstrain of the Au-LNO films when the strain state changes were almost the same as those in the LNO films, as shown in Fig. 5b. Thus, microstrain is independent of the external strain in Au-LNO films, which coincides with the observed lack of change in resistivity. 

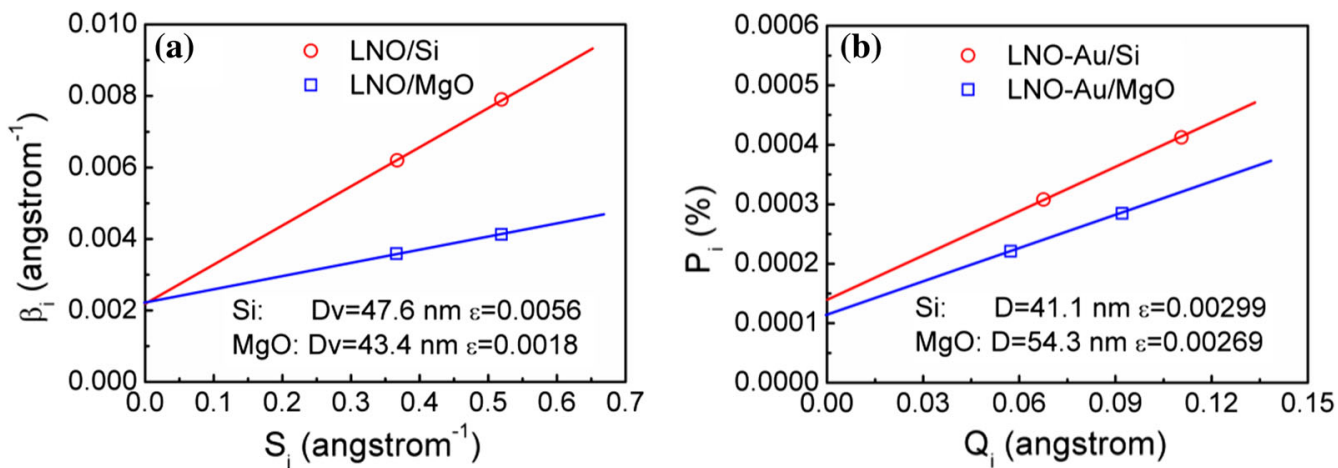

Fig. 5. Microstrain in LNO and Au-LNO films under tensile and compressive strain $\left(D\right.$ : grain size, $\varepsilon$ : microstrain strain, $\beta=\beta \cos \theta / \lambda, S_{i}=2 \sin \theta / \lambda$, $P_{i}=\beta^{2} / \operatorname{tg}^{2} \theta, Q_{i}=\lambda \beta / \operatorname{tg} \theta \sin \theta$ ). Analyses based on (a) the William-Hall method and (b) an alternative method proposed by Klug and Alexander.
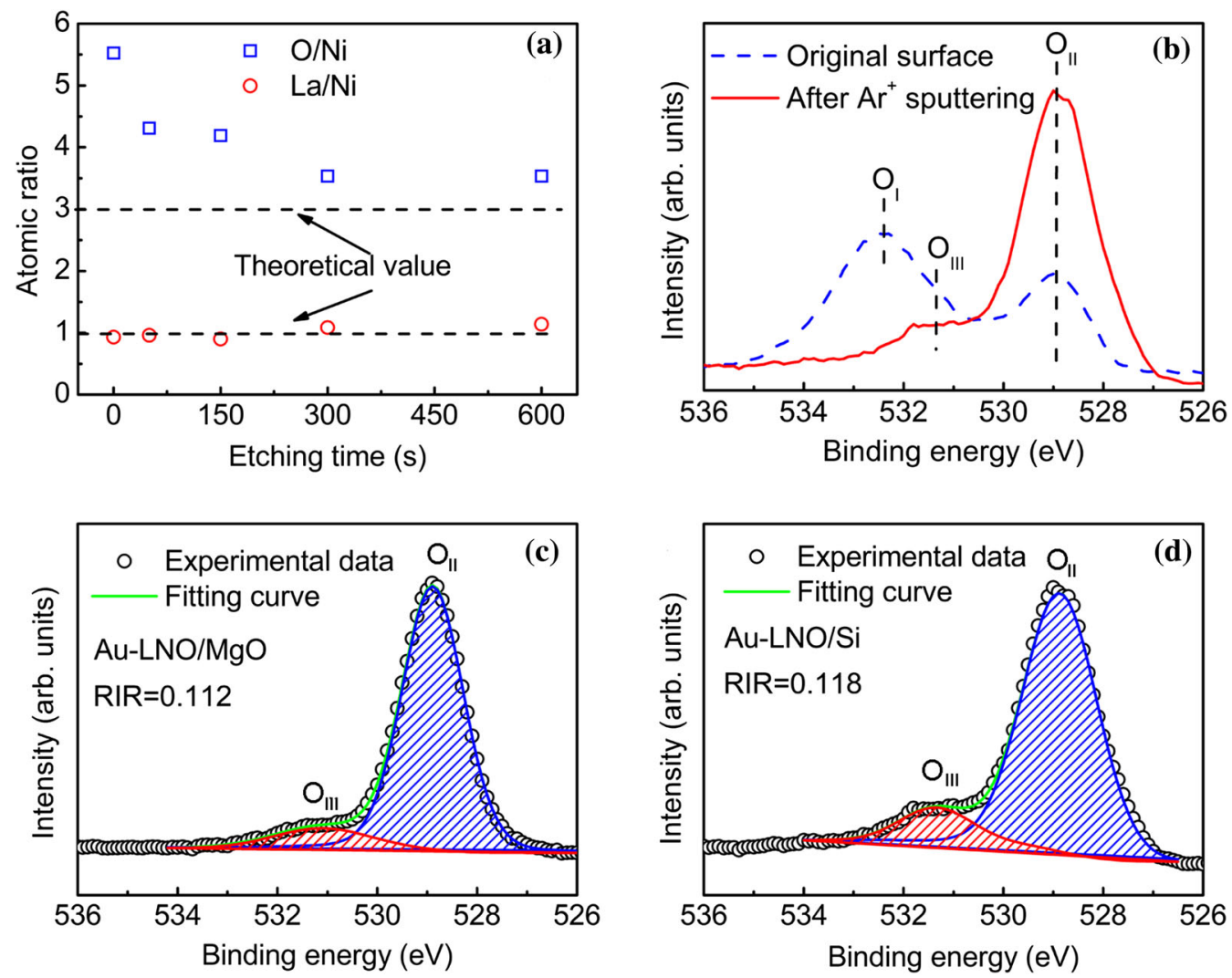

Fig. 6. XPS analysis of LNO and Au-LNO films. (a) Depth profile of the atomic ratio between La, Ni, and O in Au-LNO films on Si substrates. (b) $\mathrm{O} 1 \mathrm{~s}$ spectra of Au-LNO films before and after $\mathrm{Ar}^{+}$etching. (c) and (d) $\mathrm{O} 1 \mathrm{~s}$ spectra of $\mathrm{Au}$-LNO films in different strain states (to avoid the effects of contamination from the air, the films were etched by $\mathrm{Ar}^{+}$for $300 \mathrm{~s}$ in the vacuum chamber).

\section{Chemical Composition of Au-LNO Films}

High-valence $\mathrm{Ni}^{3+}$ is thermodynamically unstable; thus, the formation of oxygen vacancies is relatively easy. This type of defect dominates in LNO films and has a close relationship with the film strain state. ${ }^{18}$ Therefore, the microstrain in LNO films is more closely related to the concentration of oxygen vacancies than cation deficiency. Figure 6a shows a depth profile of the element ratio in the films measured by XPS. The Ni-to-La cation ratio remained constant, consistent with the theoretical value of the films. The $\mathrm{O} 1 \mathrm{~s}$ peak (Fig. $6 \mathrm{~b}$ ) could be fitted by three peaks, indicating three oxygen states in the Au-LNO films. The peak at $532.4 \mathrm{eV}\left(O_{I}\right)$ was associated with adsorbed oxygen in the films, which mainly existed on the film surfaces. The amount of adsorbed oxygen increased from the interior to the surface of the film, accounting for the large deviation of oxygen stoichiometry in Fig. 6a. The peak at $528.9 \mathrm{eV}\left(O_{I I}\right)$ was attributed to lattice oxygen in the film, and oxygen vacancies contributed to the third peak at $531.5 \mathrm{eV}\left(O_{I I I}\right){ }^{30}$ The relative density of 
oxygen vacancies was estimated from the relative intensity ratio of the peaks $\left(\mathrm{RIR}=O_{I I I} / O_{I I}\right)$. According to our previous work, the concentration of oxygen vacancies in LNO films under tensile strain was greater than that under compressive strain, which in turn had a strong effect on the electrical properties of LNO films. ${ }^{14}$ However, Fig. $6 c$ and $d$ reveal that the density of oxygen vacancies remained almost constant in Au-LNO films under both tensile and compressive strain. Therefore, tensile strain does not result in the formation of more oxygen vacancies, in contrast to the case for Au-free LNO films. This result accounts for the strain-independent resistivity of the Au-LNO films.

Consequently, the existence of microstrain rather than macrostrain is a decisive factor for the electrical properties of polycrystalline LNO films, unlike the case for epitaxial LNO films, where macrostrain dominates. The variation of microstrain in polycrystalline LNO films is attributed to change of oxygen vacancy concentration. The introduction of $\mathrm{Au}$ effectively decreases the density of oxygen vacancies by improving the crystallinity of the films and stabilizes the concentration of oxygen vacancies in the LNO films under different external strains. This effect is responsible for the minor change in the resistivities of $\mathrm{Au}-\mathrm{LNO}$ films.

\section{CONCLUSIONS}

We prepared (110)-oriented $\mathrm{Au}-\mathrm{LaNiO}_{3-\delta}$ composite films on different substrates by a sol-gel method. The effects of external strain on the electrical properties of the films were then investigated by using substrates with different mismatches between the CTEs of the films and substrates. The presence of $\mathrm{Au}$ had a more pronounced effect on the electrical properties of the LNO films than that expected by a simple combination of the properties of the two constituent phases. The introduction of $\mathrm{Au}$ decreased the resistivity of LNO films and also alleviated the dependence of their electrical properties on the strain state. The room-temperature resistivity of the AuLNO films was strain-independent, unlike that of Au-free LNO films.

For polycrystalline LNO films, microstrain rather than macrostrain in the films was the decisive factor determining their electrical properties. The variation of microstrain in the composite Au-LNO films remained almost constant, consistent with their changes in resistivity under different external strains. The microstrain was closely related to oxygen vacancy density, which affected the electrical properties of the films. The introduced $\mathrm{Au}$ acted as nuclei seeds and promoted crystallization of the films, which decreased the number of oxygen vacancies and stabilized their concentration under different external strains. This consequently weakened the strain dependence of the room-temperature resistivity of the LNO films.

\section{ACKNOWLEDGMENTS}

This work was financially supported by the National Natural Science of Foundation of China (nos. 51202256 and 51571057) and the Fundamental Research Funds for the Central Universities (no. N170204012).

\section{REFERENCES}

1. P.D.C. King, H.I. Wei, Y.F. Nie, M. Uchida, C. Adamo, S. Zhu, X. He, I. Bozovic, D.G. Schlom, and K.M. Shen, Nat. Nanotechnol. 9, 443 (2014).

2. H.H. Chen, A.J. Millis, and C.A. Marianetti, Phys. Rev. Lett. 111, 116403 (2013).

3. B. Geisler and R. Pentcheva, Phys. Rev. Mater. 2, 055403 (2018).

4. X.X. Huang, X.G. Tang, J.L. Lai, Y.P. Jiang, Q.X. Liu, and D.P. Xiong, J. Electron. Mater. 10, 3783 (2015).

5. R. Li, X.G. Tang, Y.P. Jiang, Y.K.Q.X. Liu, and L.L. Jiang, J. Mater. Sci.: Mater. Electron. 28, 12646 (2017).

6. K. Tsubouchi, I. Ohkubo, H. Kumigashira, Y. Masumoto, T. Ohnishi, M. Lippmaa, H. Koinuma, and M. Oshima, Appl. Phys. Lett. 92, 262109 (2008).

7. L. Qiao and X.F. Bi, Acta Mater. 57, 4109 (2009).

8. E.J. Moon, J.M. Rondinelli, N. Prasai, B.A. Gray, M. Kareev, J. Chakhalian, and J.L. Cohn, Phys. Rev. B 85, 121106 (2012).

9. M. Wu, E. Benckiser, M.W. Haverkort, A. Frano, Y. Lu, U. Nwankwo, S. Brueck, P. Audehm, E. Goering, S. Macke, V. Hinkov, P. Wochner, G. Christiani, S. Heinze, G. Logvenov, H.U. Habermeier, and B. Keimer, Phys. Rev. B 88, 125124 (2013).

10. S.J. May, J.W. Kim, J.M. Rondinelli, E. Karapetrova, N.A. Spaldin, A. Bhattacharya, and P.J. Ryan, Phys. Rev. B 82, 014110 (2010).

11. G.S. Wang, D. Remiens, E. Dogheche, and X.L. Dong, Appl. Phys. A 88, 657 (2007).

12. G. Bai, Z.G. Liu, X.B. Yan, and C.C. Zhang, J. Appl. Phys. 116, 054103 (2014).

13. B.L. Peng, Q. Zhang, Y.N. Lyu, L.J. Liu, X.J. Lou, C. Shaw, H.T. Huang, and Z.L. Wang, Nano Energy 47, 285 (2018).

14. M.W. Zhu, H.L. Wang, H. Lei, Y.J. Zhang, N. Jia, and Z.J. Wang, Appl. Phys. A 122, 364 (2016).

15. D. Misra and T.K. Kundu, J. Electron. Mater. 46, 150 (2017),

16. M.W. Zhu, P. Komissinskiy, A. Radetinac, Z.J. Wang, and L. Alff, J. Appl. Phys. 117, 155306 (2015).

17. J.D. Sayre, K.T. Delaney, N.A. Spaldin, Interplay between strain and oxygen vacancies in lanthanum aluminate. (arXiv by Cornell Univerisity, 2012), https://arxiv.org/abs/1202. 1431v1. Accessed 7 Feb. 2012.

18. F. Conchon, A. Boulle, R. Guinebretiere, C. Girardot, S. Pignard, J. Kreisel, F. Weiss, E. Dooryhee, and J. Hodeau, Appl. Phys. Lett. 91, 192110 (2007).

19. B.A. Akgun, C. Durucan, and N.P. Mellott, J. Sol Gel. Sci. Technol. 58, 277 (2011).

20. X.K. Ning, Z.J. Wang, and Z.D. Zhang, Adv. Funct. Mater. 24, 5393 (2014).

21. N. Jia, Y.D. Wang, S.D. Wu, W.Z. Han, Y.N. Wang, J.N. Deng, and P.K. Liaw, Script Mater. 54, 1247 (2006).

22. Z. Zhang, F. Zhou, and E.J. Lavernia, Metall. Mater. Trans. A 34A, 1349 (2003).

23. H.P. Klug and L.E. Alexander, $X$-ray diffraction procedures for polycrystalline and amorphous materials, 2nd ed. (New York: Wiley, 1974), pp. 618-687.

24. M.F. Ashby and Y.J.M. Brechet, Acta Mater. 51, 5801 (2003).

25. J. Son, P. Moetakef, J.M. Lebeau, D. Ouellette, L. Balents, S.J. Allen, and S. Stemmer, Appl. Phys. Lett. 96, 062114 (2010).

26. K. Horiba, R. Eguchi, M. Taguchi, A. Chainani, A. Kikkawa, Y. Senba, H. Ohashi, and S. Shin, Phys. Rev. B 76, 155104 (2007). 
27. J.J. Zhang, H. Zheng, Y. Ren, and J.F. Mitchell, Cryst. Growth Des. 17, 2730 (2017).

28. N. Gayathri, A.K. Raychaudhuri, X.Q. Xu, J.L. Peng, and R.L. Greene, J. Phys.: Condens. Matter 10, 1323 (1998).

29. Y.D. Wang, R. Lin Peng, X.L. Wang, and R.L. McGreevy, Acta Mater. 50, 1717 (2002).
30. L. Qiao and X.F. Bi, Thin Solid Films 519, 943 (2010).

Publisher's Note Springer Nature remains neutral with regard to jurisdictional claims in published maps and institutional affiliations. 